\title{
Renal Outcome in Patients Undergoing Cardiac Surgery Using Cardiopulmonary Bypass
}

Mohammed S. Alqarni ${ }^{1}$, Abdullah H. Ghunaim ${ }^{2}$, Abdulkarim W. Abukhodair ${ }^{1}$, Jose Andres Fernandez ${ }^{3}$, Sean R. Bennett ${ }^{3}$

1. Medicine, King Saud Bin Abdulaziz University for Health Sciences, Jeddah, SAU 2. Medicine, College of Medicine, King Abdulaziz University, Jeddah, SAU 3. Anesthesiology, King Faisal Cardiac Center, King Abdullah Medical City, Jeddah, SAU

Corresponding author: Sean R. Bennett, seanbennett0709@gmail.com

\section{Abstract \\ Introduction}

Renal dysfunction is a significant variable in determining the outcome of surgery, such as cardiopulmonary bypass graft and valvular replacement, used to treat cardiovascular diseases. In Saudi Arabia, the incidence of renal failure and diabetes is higher than in most western populations. Our aim is to determine the renal outcome of patients who underwent cardiac surgery at King Faisal Cardiac Center from 2014 to 2017.

\section{Methods}

This a retrospective cohort study using a non-probability consecutive sampling technique for selection of the study population to assess the renal outcome in cardiac surgery patients using cardiopulmonary bypass from May 2014 to June 2017 in King Faisal Cardiac Center, Jeddah. Patients older than 18 years of age undergoing cardiac surgery, with available data, were included. Categorical variables were summarized by percentages and frequencies, and continuous variables by means and standard deviations, or medians and interquartile ranges if their distributions were skewed. Logistic regression was done with post-op renal impairment as the dependent variable and pre-op renal dysfunction, age, gender, smoking status, diabetes, hypertension, dyslipidemia, and cardiopulmonary bypass time as independent variables.

\section{Results}

Our sample size included 244 patients who underwent cardiac surgery in this study period; their mean age was $60.5(\mathrm{SD}=7.5)$ with a mean body mass index (BMI) of $28.62(\mathrm{SD}=5.19)$. Among our population, $73 \%(\mathrm{n}=$ $179)$ were males and $27 \%(n=66)$ were females. Two percent $(2 \%)$ of patients $(n=5)$ died within 30 days, $4 \%$ of patients $(n=10)$ with temporary dialysis, $8 \%$ of patients $(n=19)$ with postoperative renal dysfunction, and no patients with permanent dialysis. The data showed a significant relationship between levels of creatinine preoperatively and postoperative renal dysfunction ( $\mathrm{p}$-value $=0.0001, \mathrm{OR}=1.05,95 \% \mathrm{CI}$ of 1.031 to 1.064$)$.

Received 06/27/2020

Review began 06/30/2020 Review ended 06/30/2020 Published 07/06/2020

\section{(c) Copyright 2020}

Alqarni et al. This is an open access article distributed under the terms of the Creative Commons Attribution License CC-BY 4.0., which permits unrestricted use, distribution, and reproduction in any medium, provided the original author and source are credited.

\section{Conclusion}

The main predictor of poor renal outcomes for cardiac surgery is preoperative creatinine. While other factors, such as age, gender, body mass index, cardiopulmonary bypass time, diabetes, hypertension, and dyslipidemia, did not show any risk to the postoperative renal outcome.

Categories: Anesthesiology, Cardiac/Thoracic/Vascular Surgery, Nephrology

Keywords: renal outcome, cardiac surgery, cardiopulmonary bypass, kingdom of saudi arabia (ksa)

\section{Introduction}

According to the World Health Organization (WHO), in 2017, more than 31\% of the deaths in the world (17.9 million people) were due to cardiovascular disease [1]. Cardiovascular diseases are common in the population due to the increased amount of modifiable risk factors, such as high blood pressure, dyslipidemia, smoking, diabetes mellitus, obesity, and chronic kidney disease (CKD), and non-modifiable risk factors such as age, family history and gender [2-3]. These risk factors increase the risk of getting these diseases and could affect severity [4-5].

Renal dysfunction is also a major risk factor for end-stage renal failure and premature death [6]. A paper in 2010 calculated the global prevalence of CKD to be around 497 million adults in the world, which clearly proves that this is a global problem [7]. In addition, according to the Global Burden of Disease Study 2013, there was a $134.6 \%$ increase in the mortality of CKD patients since 1990 , with a staggering 956,200 deaths in 2013 alone [8]. 
Renal dysfunction is a significant variable in determining the outcome of the surgeries used to treat these cardiovascular diseases - the most common surgeries being cardiopulmonary bypass graft (CABG) and valvular replacement (VR) [9]. Up to $30 \%$ mortality has been reported [10]. According to the literature, the severity of renal dysfunction also affects mortality [10-11]. In Saudi Arabia, the incidence of renal failure and diabetes is higher than in most western populations [12-13]. The King Faisal Cardiac Center (KFCC) at the National Guard Hospital Jeddah recently started cardiac surgery. Our aim is to determine the renal outcome of patients who underwent cardiac surgery at KFCC from 2014 to 2017.

\section{Materials And Methods}

This a retrospective cohort study using a non-probability consecutive sampling technique for the selection of the study population to assess the renal outcome in cardiac surgery patients using cardiopulmonary bypass from May 2014 to June 2017 in KFCC, Jeddah. The research project was approved by King Abdullah International Medical Research Center, Jeddah, Saudi Arabia (KAIMRC) and the Institutional Review Board (IRB). Our data was collected from hard files and soft files (BestCare, ezCaretech, Jung-gu, Seoul) at medical records. Patients older than 18 years of age who were undergoing cardiac surgery with available data were included. The data collection sheet included the following demographics: age, gender, body mass index (BMI), type of cardiac surgery, co-morbidities such as diabetes mellitus, hypertension, and hyperlipemia. Also, preoperative serum creatinine levels, preoperative renal dysfunction, and cardiopulmonary bypass (CPB) time were also included as independent variables. The main outcomes were 30-day mortality, new dialysis, and postoperative renal dysfunction. Acute renal dysfunction is defined as serum creatinine levels greater than or equal to $200 \mu \mathrm{mol} / \mathrm{L}[14]$.

Categorical variables were summarized by percentages and frequencies, and continuous variables by means and standard deviations or medians and interquartile ranges if their distributions were skewed. Baseline univariate comparisons between postoperative renal dysfunction and no postoperative renal dysfunction were made with Wilcoxon test rank-sum and the chi-square tests where appropriate. Logistic regression was done with postop renal dysfunction as the dependent variable and preop renal dysfunction, age, gender, smoking status, diabetes, hypertension, dyslipidemia, and CPB time as independent variables. Independent variables with $\mathrm{p}$-value $<0.05$ were considered significant. All results were computed using IBM SPSS version 23 (IBM Corp., Armonk, NY).

\section{Results}

Our sample size included 244 patients who underwent cardiac surgery in this study period; their mean age was $60.5(\mathrm{SD}=7.5)$ with a mean BMI of $28.62(\mathrm{SD}=5.19)$. Among our population, $73 \%(\mathrm{n}=179)$ were males and $27 \%(n=66)$ were females. Co-morbidities included hypertension $80 \%(n=197)$, diabetes $77 \%(n=190)$, dyslipidemia $64 \%(n=156)$, and smoking $27 \%(n=65)$. The patients in this sample displayed a median 77 $\mu \mathrm{mol} / \mathrm{L}(\mathrm{IQR}=66$ - 99.75) of postoperative creatinine and a median of 117 minutes (IQR = 84-143) for bypass time. All the characteristics of the patients are summarized in Table 1 and Figure 1. 


\section{Cureus}

Patient characteristic

$n=244$ patients

Preoperative characteristics

Age (years)

Mean \pm S.D.

$60.5 \pm 7.5$

Male gender \%

$73 \%$

BMI $\left(\mathrm{kg} / \mathrm{m}^{2}\right)$

Mean \pm S.D.

$28.62 \pm 5.19$

Smoking \%

$27 \%$

Hypertension \%

$80 \%$

Diabetes mellitus \%

$77 \%$

Dyslipidemia \%

$64 \%$

Preop creatinine $(\mu \mathrm{mol} / \mathrm{L})$

Median \pm IQR

$83 \pm 31$

Intraoperative characteristics

Type of surgery (\%)

CABG

$73 \%$

Valve

$17 \%$

CABG + Valve

$8 \%$

Other

$2 \%$

CPB time in minutes

Median \pm IQR

$117 \pm 59$

Postoperative characteristics

Postop creatinine $(\mu \mathrm{mol} / \mathrm{L})$

Median \pm IQR

$77 \pm 34$

Temporary dialysis \% (n)

$4 \%(10)$

Postoperative renal dysfunction \% (n)

$8 \%(19)$

Mortality \% (n)

$2 \%(5)$

\section{TABLE 1: Patients' characteristics}

BMI: Body Mass Index, CABG: Coronary Arteries Bypass Graft, VR: Valvular Replacement, CPB: Cardiopulmonary Bypass, S.D: Standard Deviation, IQR: Interquartile Range 


\section{Cureus}

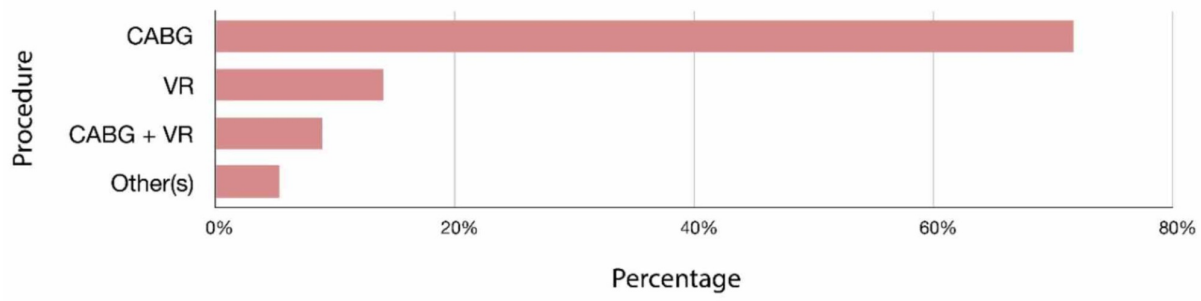

\section{FIGURE 1: Types of procedures}

CABG: Coronary Arteries Bypass Graft, VR: Valvular Replacement

There were $2 \%$ of patients $(n=5)$ who died within 30 days, $4 \%$ of patients $(n=10)$ with temporary dialysis, $8 \%$ of patients $(n=19)$ with postoperative renal dysfunction, and no patients with permanent

dialysis. Twenty-five patients had postoperative renal dysfunction, 19 of these had preop renal dysfunction. The data showed a significant relationship between the levels of creatinine preoperatively and postoperative renal dysfunction $(\mathrm{p}$-value $=0.0001, \mathrm{OR}=1.05,95 \% \mathrm{CI}$ of 1.031 to 1.064 ), as shown in Figure2.

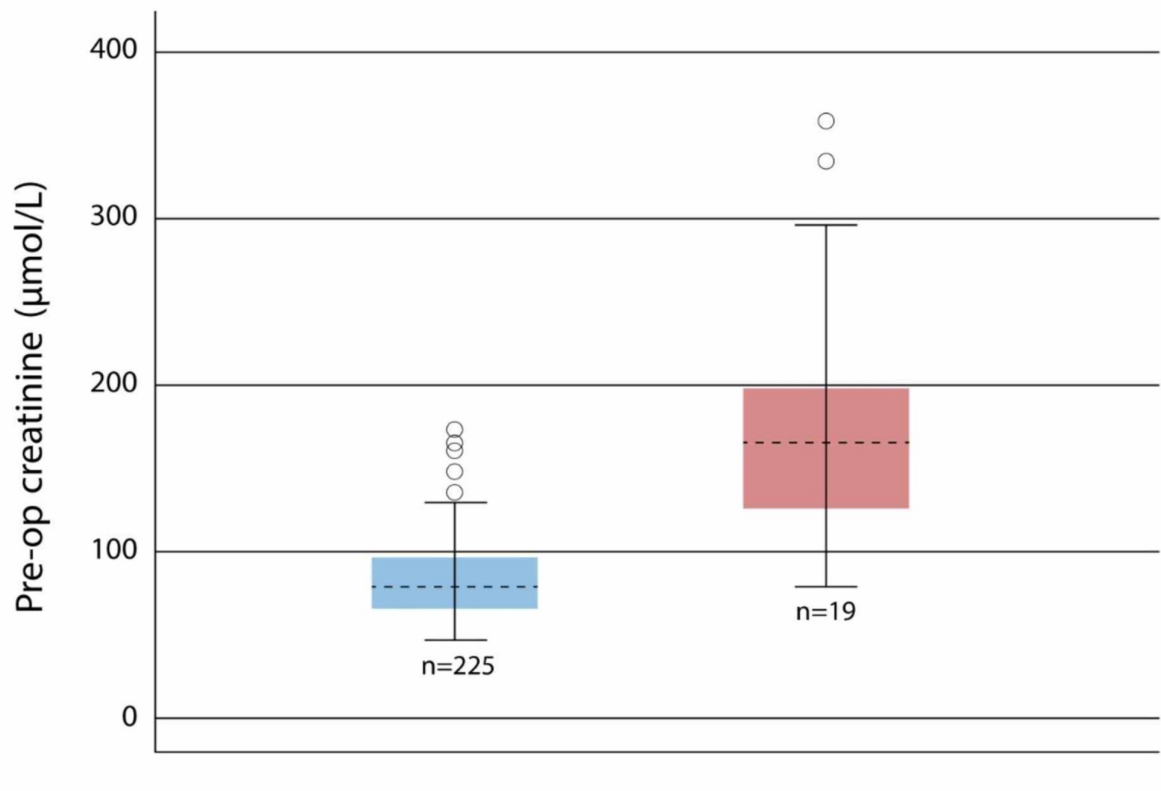

$\mathrm{Cr}<200 \mu \mathrm{mol} / \mathrm{L} \quad \mathrm{Cr}>200 \mu \mathrm{mol} / \mathrm{L}$

Post-op renal function

FIGURE 2: Preoperative creatinine in patients with postoperative renal dysfunction vs patients with no postoperative renal dysfunction

CPB time $(\mathrm{p}$-value $=0.788)$, hypertension $(\mathrm{p}$-value $=0.619)$, diabetes $(\mathrm{p}$-value $=0.961)$, and age $(\mathrm{p}$-value $=$ 0.180 ) were not statistically significant difference (Figure 3 and Table 2 ). 


\section{Cureus}

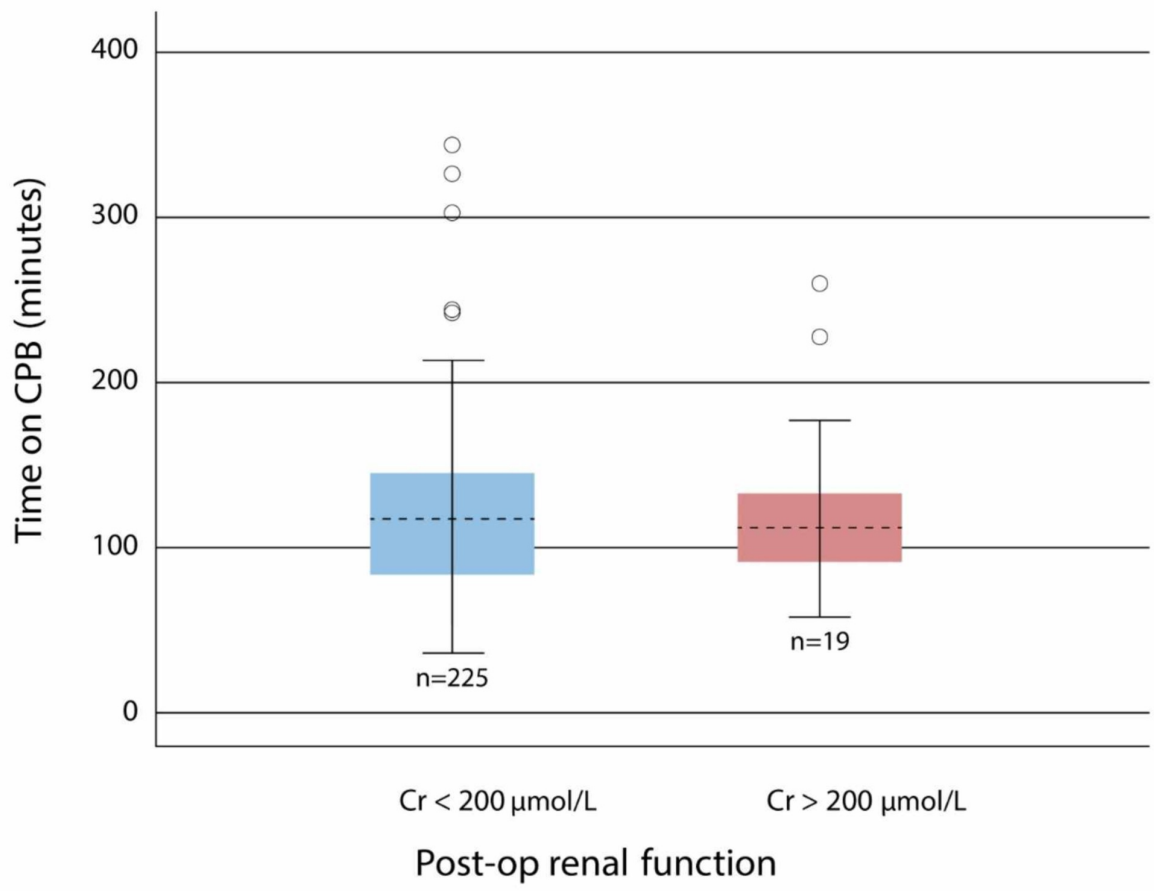

FIGURE 3: Time on CPB in patients with postoperative renal dysfunction vs patients with no postoperative renal dysfunction

CPB: cardiopulmonary bypass

\begin{tabular}{|l|l|l|l|l|l|l|l|l|}
\hline & B & S.E. & Wald & df & Significance & Exp(B) & Lower C.I. for EXP(B) & Upper C.I. for EXP(B) \\
\hline Creatinine & .048 & .009 & 26.823 & 1 & & 1.050 & 1.031 & 1.069 \\
Time on bypass & -.002 & .007 & .072 & 1 & .788 & .998 & .984 & 1.012 \\
Hypertension & .678 & 1.363 & .247 & 1 & .619 & 1.969 & .136 & 28.458 \\
Age & .050 & .038 & 1.801 & 1 & .180 & 1.052 & .977 & 1.132 \\
Diabetes & .060 & 1.228 & .002 & 1 & .961 & 1.061 & .096 & 11.784 \\
\hline
\end{tabular}

TABLE 2: Independent predictors of postoperative renal dysfunction

B: Base, S.E.: Standard Error, S.D.: Standard Deviation, Wald: Wald Chi-Squared Test, df: degrees of freedom, C.I.: Confidence Interval, Exp: Natural Exponential Function

\section{Discussion}

CPB time has been associated with postoperative renal dysfunction in recent studies; however, our study did not show any statistical significance [15-16]. We suspect that the low sample size was the main reason that we got an insignificant association between postoperative renal dysfunction and CPB time. Our study has shown a significant association with preoperative renal function and postoperative renal dysfunction, and this is similar to many studies that show that preoperative renal dysfunction, as measured by serum creatinine levels, increases the risk of developing acute kidney injury [17-18].

Mortality after surgery, as well as postoperative temporary dialysis, was low. Also, none of the participants required permanent dialysis, and only $8 \%$ of our population showed postoperative renal dysfunction. In contrast to the other articles that showed a higher incidence of mortality, dialysis, and renal dysfunction [16-18].

There were more males compared to females, similar to the results from different articles that have been conducted with a male-to-female ratio of almost 3:1 [16-19]. Demographic data and cardiac co-morbidities, 
such as hypertension, diabetes, and dyslipidemia, did not show any statistically significant association with postoperative renal dysfunction. Likewise, a study that was done in Brazil concluded that there was no association between these co-morbidities and acute kidney injury after undergoing cardiac surgery [20].

\section{Conclusions}

The main predictor of poor renal outcome after cardiac surgery is preoperative creatinine, while other variables, such as age, gender, BMI, CPB time, diabetes, hypertension, and dyslipidemia did not show any risk to the renal outcome. These results deviate toward a trend that resembles recent literature; however, a prospective cohort with a larger sample size could show statistical significance in regard to CPB.

\section{Additional Information}

\section{Disclosures}

Human subjects: All authors have confirmed that this study did not involve human participants or tissue. Animal subjects: All authors have confirmed that this study did not involve animal subjects or tissue. Conflicts of interest: In compliance with the ICMJE uniform disclosure form, all authors declare the following: Payment/services info: All authors have declared that no financial support was received from any organization for the submitted work. Financial relationships: All authors have declared that they have no financial relationships at present or within the previous three years with any organizations that might have an interest in the submitted work. Other relationships: All authors have declared that there are no other relationships or activities that could appear to have influenced the submitted work.

\section{References}

1. World Health Organization. The top 10 causes of death . (2017). https://www.who.int/en/news-room/factsheets/detail/cardiovascular-diseases-(cvds).

2. Gordon T, Kannel WB: Multiple risk functions for predicting coronary heart disease: the concept, accuracy, and application. Am Heart J. 1982, 103:1031-1039. 10.1016/0002-8703(82)90567-1

3. Kannel WB, McGee DL: Diabetes and glucose tolerance as risk factors for cardiovascular disease: the Framingham study. Diabetes Care. 1979, 2:120-126. 10.2337/diacare.2.2.120

4. Gordon T, Castelli WP, Hjortland MC, Kannel WB, Dawber TR: Diabetes, blood lipids, and the role of obesity in coronary heart disease risk for women. The Framingham Study. Ann Int Med. 1977, 87:393-397. 10.7326/0003-4819-87-4-393

5. Sarnak MJ, Levey AS, Schoolwerth AC, et al.: Kidney disease as a risk factor for development of cardiovascular disease. Circulation. 2003, 28:2154-2169. 10.1161/01.CIR.0000095676.90936.80

6. Jha V, Garcia-Garcia G, Iseki K, et al.: Chronic kidney disease: global dimension and perspectives . Lancet. 2013 Jul, 26:260-272. 10.1016/S0140-6736(13)60687-X

7. Mills KT, Xu Y, Zhang W, et al.: A systematic analysis of worldwide population-based data on the global burden of chronic kidney disease in 2010. Kidney Int. 2015, 30:950-957. 10.1038/ki.2015.230

8. Naghavi M, Wang H, Lozano R, Davis A, Liang X, Zhou M: GBD 2013 Mortality and Causes of Death Collaborators. Global, regional, and national age-sex specific all-cause and cause-specific mortality for 240 causes of death, 1990-2013: a systematic analysis for the Global Burden of Disease Study 2013. Lancet. 2015, 10:117-171. 10.1016/S0140-6736(14)61682-2

9. Durmaz I, Büket S, Atay Y, et al.: Cardiac surgery with cardiopulmonary bypass in patients with chronic renal failure. J Thorac Cardiovasc Surg. 1999, 118:306-315. 10.1016/S0022-5223(99)70221-7

10. Frenken M, Krian A: Cardiovascular operations in patients with dialysis-dependent renal failure . Ann Thorac Surg. 1999, 68:887-893. 10.1016/S0003-4975(99)00554-8

11. Chen S-W, Chang C-H, Lin Y-S, et al.: Effect of dialysis dependence and duration on post-coronary artery bypass grafting outcomes in patients with chronic kidney disease: a nationwide cohort study in Asia. Int J Cardiol. 2016, 223:65-71. 10.1016/j.ijcard.2016.08.121

12. King H, Aubert RE, Herman WH: Global burden of diabetes, 1995-2025: prevalence, numerical estimates, and projections. Diabetes Care. 1998, 21:1414-1431. 10.2337/diacare.21.9.1414

13. Al-Nozha MM, Al-Maatouq MA, Al-Mazrou YY, Al-Harthi SS: Diabetes mellitus in Saudi Arabia . Saudi Med J. 2004, 24:1603-1610.

14. Bellomo R, Ronco C, Kellum JA, Mehta RL, Palevsky P: Acute renal failure - definition, outcome measures, animal models, fluid therapy and information technology needs: the Second International Consensus Conference of the Acute Dialysis Quality Initiative (ADQI) Group. Crit Care. 2004, 8:204-212. $10.1186 /$ cc2872

15. Bellomo R, Auriemma S, Fabbri A, et al.: The pathophysiology of cardiac surgery-associated acute kidney injury (CSA-AKI). Int J Artif Organs. 2008, 31:166-78. 10.1177/039139880803100210

16. Axtell AL, Fiedler AG, Melnitchouk S, et al.: Correlation of cardiopulmonary bypass duration with acute renal failure after cardiac surgery. J Thorac Cardiovasc Surg. 2020, 159:170-178. 10.1016/j.jtcvs.2019.01.072

17. Jayavelan R, Gopinathan G, Kavin K, Shanmugasundarama R, Stephen MG, Pragasam AC, Thangavel P: Effects of cardiopulmonary bypass perfusion temperature on perioperative renal function in adult patients undergoing cardiac surgery. J Saudi Heart Assoc. 2019, [Epub ahead of print]: 10.1016/j.jsha.2019.09.004

18. Husain-Syed F, Ferrari F, Sharma A, et al.: Preoperative renal functional reserve predicts risk of acute kidney injury after cardiac operation. Ann Thorac Sur. 2018, 4:1094-1101. 10.1016/j.athoracsur.2017.12.034

19. Salis S, Mazzanti VV, Merli G, Salvi L, Tedesco CC, Veglia F, Sisillo E: Cardiopulmonary bypass duration is an independent predictor of morbidity and mortality after cardiac surgery. J Cardiothorac Vasc Anesth. 2008, 22:814-822. 10.1053/j.jvca.2008.08.004

20. Rodrigues AJ, Barbosa Evora PR, Bassetto S, Alves L Júnior, Filho AS, Ferreira Araújo W, Andrade Vicent WV: 


\section{Cureus}

Risk factors for acute kidney injury after cardiac surgery [Article in Portuguese] . Rev Bras Cir Cardiovasc 2019, 24:441-446. 10.1590/S0102-76382009000500003 